\title{
Targeted Immunotherapy Strategies in ANCA-Associated Vasculitis
}

Xavier Puéchal ${ }^{1,2,3}$

${ }^{1}$ Centre de Référence des Maladies Systémiques Auto-immunes rares, Département de

Médecine Interne, Hôpital Cochin, Assistance Publique - Hôpitaux de Paris, 27, rue du faubourg Saint Jacques, 75014 Paris, France

${ }^{2}$ Université Paris Descartes, 12 Rue de l'École de Médecine, 75006 Paris, France

${ }^{3}$ Institut Cochin, INSERM U1016, CNRS UMR 8104, Paris, France

Tel.: +33 158413 241; Fax: +33158412968

E-mail: xavier.puechal@aphp.fr 


\section{ABSTRACT}

Targeted immunotherapy is substantially improving the management of ANCA-associated vasculitides (AAV), which include granulomatosis with polyangiitis (GPA, Wegener's granulomatosis), microscopic polyangiitis (MPA), and eosinophilic granulomatosis with polyangiitis (EGPA, Churg-Strauss syndrome). This article reviews the current role for targeted immunotherapy in AAV, its validated indications, and avenues for further development. Rituximab is a validated induction treatment for GPA and severe MPA. Rituximab in these indications is not less effective than cyclophosphamide and is particularly useful in patients with refractory or relapsing disease, women of childbearing potential, and patients previously treated with cyclophosphamide. Rituximab is more effective than cyclophosphamide for treating relapses. For remission maintenance therapy, which is indispensable, rituximab has been proven superior over conventional immunosuppressive treatment. Rituximab is licensed in the USA and in Europe for the induction treatment of severe forms of GPA and MPA. An extension study for remission maintenance therapy is ongoing. In EGPA, although maintenance treatment with the interleukin-5 antagonist mepolizumab is effective in decreasing glucocorticoid requirements and in alleviating asthma and sinonasal symptoms, its efficacy on the vasculitis remains somewhat unclear. Mepolizumab is licensed for use in EGPA, and rituximab is also being evaluated as an induction and maintenance agent. Immunoglobulins can be helpful as an adjuvant treatment for active AAV with severe immunodepression, notably when infections occur. Plasma exchange is indicated in AAV with advanced renal dysfunction and, perhaps, in the event of alveolar hemorrhage, a possibility that will be assessed in 2018 in a large international study. 
Keywords: Therapeutic immunomodulation. Necrotizing vasculitis. Granulomatosis with polyangiitis. Eosinophilic granulomatosis with polyangiitis. Biological agents. Rituximab. 


\section{Principles of the treatment of ANCA-associated vasculitis}

\section{(AAV)}

\subsection{Granulomatosis with polyangiitis (GPA)}

Granulomatosis with polyangiitis (GPA, Wegener's granulomatosis) requires induction therapy with a combination of high-dose glucocorticoids and immunosuppressants (cyclophosphamide or rituximab or, in limited forms, methotrexate) to obtain a remission $(1,2)$. As soon as this goal is achieved, the patient is switched to remission maintenance therapy, which classically consisted in azathioprine or methotrexate (1-4). Prolonged treatment is needed to control the disease. Despite maintenance therapy, over half the patients experience relapses (4). One consequence is a high incidence of severe adverse events.

\subsection{Microscopic polyangiitis (MPA)}

A treatment strategy must be designed in a specialized department, based on disease severity as assessed by the Five-Factor Score (FFS) $(2,5)$ (Table 1). Induction therapy to obtain a remission relies chiefly on glucocorticoids $(2,6)$, which are combined with immunosuppressants if the FFS is 1 or higher $(2,5,7)$. Remission maintenance therapy is then started, classically using azathioprine or methotrexate if the FFS is 1 or higher (2-4).

\section{B-cell targeted therapies}




\subsection{Rationale for targeting $B$ cells}

Many lines of evidence support the use of biotherapies targeting the B cell in patients with AAV. Both B-cell activation and the level of B-cell-activating factor (BAFF) correlate with disease activity $(8,9)$. Granulomas contain foci characterized by an abundance of $\mathrm{CD} 20^{+} \mathrm{B}$ cells including autoreactive memory B cells that exhibit affinity for the PR3 antigen of antineutrophil cytoplasmic antibodies (ANCAs) and secrete autoantibodies $(10,11)$. ANCAs are known to be involved in the pathogenesis of AAV (12). In addition, ANCAs stimulate neutrophils to release BAFF, which may then perpetuate the production of ANCA by increasing the survival of autoreactive B cells (13).

\subsection{Rituximab}

Rituximab is a chimeric monoclonal antibody to the CD20 receptor on the surface of B cells. CD20 is highly specific of mature B cells and is absent from B-cell precursors and plasma cells. By binding to CD20, rituximab destroys the B cells.

\subsubsection{Rituximab for induction therapy}

Rituximab is an option for the induction treatment of AAV. Rituximab was licensed in the USA in April 2011, in combination with glucocorticoids, for the treatment of GPA and MPA in adults. In March 2013, rituximab was licensed in Europe, in combination with glucocorticoids, for severe GPA and MPA.

The preliminary open-label studies were conducted in patients with GPA, most of whom had refractory disease $(14,15)$. The complete remission rate was greater than $75 \%$. The time to maximal effect was up to 3 months. Granulomatous forms responded less well than did vasculitic forms (16). 
Two multicenter randomized controlled trials compared rituximab (4 infusions of $375 \mathrm{mg} / \mathrm{m}^{2}$ at 1-week intervals) to cyclophosphamide, combined with glucocorticoid therapy, for the induction treatment of GPA or MPA. The remission rates were similar with the two medications, as was the short-term safety profile $(17,18)$. However, for the treatment of relapses, rituximab was associated with a higher remission rate (18). Patients randomized to cyclophosphamide who failed to respond to this drug were then switched to rituximab, which provided a remission in most of these cases of refractory vasculitis (19). A post hoc analysis suggested that patients with PR3-ANCA may respond better to rituximab compared to patients with ANCA to myeloperoxidase (MPO-ANCA) (20). The rate of remissions lasting at least 18 months was not lower in patients given only four weekly rituximab infusions compared to those given cyclophosphamide followed, once a remission was achieved, by azathioprine as maintenance therapy for 12 to 15 months (21). In the extension phases of these trials, the adverse event rates were comparable after 18 months between the two treatment arms. Rituximab is also effective for obtaining a new remission after a relapse in patients whose first remission was induced by rituximab $(22,23)$.

Both randomized controlled trials included only a limited number of patients with MPA $(17,18)$. The remission rate was not different between the two medications, and neither was the short-term safety profile.

In everyday practice, rituximab for induction and maintenance therapy has also produced excellent outcomes in GPA (24). After 2 years, the treatment continuation rate was $78 \%$ and the relapse-free survival rate was $85 \%$. Furthermore, the safety profile was satisfactory, with severe infection and severe adverse event rates of 4.9 and 8.1/100 patientyears, respectively.

Rituximab for induction therapy also constitutes a therapeutic advance in patients with severe GPA or MPA, notably those with relapsing or refractory disease, as well as in women 
of childbearing potential. Furthermore, rituximab therapy eliminates the need for high cumulative cyclophosphamide dosages, which are associated with increased risks of lymphoma and urinary bladder tumors.

\subsubsection{Rituximab for remission maintenance therapy}

Remission maintenance therapy is indispensable once a remission has been achieved using rituximab $(25,26)$. The multicenter open-label randomized controlled trial MAINRITSAN conducted by the French Vasculitis Research Group (Groupe Français d'Etude des Vascularites, GFEV) established the efficacy of rituximab as remission maintenance therapy in patients who had newly diagnosed or relapsing GPA or MPA with a cyclophosphamide-induced remission. Low-dose $(500 \mathrm{mg})$ rituximab infusions given at 6month intervals led to a 6-fold decrease in the risk of severe relapse within 28 months compared to conventional maintenance therapy with azathioprine for 22 months, with no difference in safety (27). In addition, 5-year follow-up data showed that rituximab was more effective in preventing relapses than was conventional initial azathioprine therapy, with no difference in safety and with better survival (28). The results of the MAINRITSAN trial have led to a license application for rituximab as remission maintenance therapy in GPA and MPA, which is currently under examination.

Nevertheless, delayed relapses have been reported after maintenance rituximab therapy. Risk factors for relapse consist of GPA (21), previous relapse (21), initially positive tests for PR3-ANCA $(21,28)$, and persistently positive tests for ANCA (28). The potential benefits of prolonging rituximab maintenance therapy are being evaluated by the GFEV and will be particularly important to assess in patients at high risk for relapse with current or past positive tests for PR3-ANCA. 
Another international randomized controlled trial is under way to determine whether rituximab ( $1 \mathrm{~g}$ at 4-month intervals) is more effective than azathioprine for remission maintenance in patients with a rituximab-induced remission of GPA or MPA (23).

Comparative studies have sought to determine the optimal rituximab strategy for remission maintenance. Although monitoring B-cell recovery or ANCA titer ascension after rituximab therapy fails to reliably predict relapses, combining both markers can be useful to determine whether re-treatment is appropriate in the individual patient $(29,30)$. The multicenter randomized controlled trial MAINRITSAN 2 conducted by the GFEV found similar relapse rates when rituximab was given either on a fixed 6-monthly schedule or only in the event of an ANCA titer increase or B-cell recovery, although the number of rituximab infusions was lower with the latter strategy (median, 3 versus 5) (30).

\subsubsection{Adverse effects of rituximab}

Rituximab is associated with adverse events, which are dominated by infections (15). Pneumocystis jiroveci pneumonia has been reported, and prophylactic therapy is recommended throughout rituximab therapy and subsequently until B-cell recovery (31). Progressive multifocal leukoencephalopathy (PML) has occurred in a few patients (32), as well as hepatitis B reactivation. No evidence exists that rituximab is associated with an increased risk of solid cancer. On the opposite, in patients given induction therapy for AAV, those given rituximab may be at lower risk for cancer than those given cyclophosphamide (33). Rituximab may induce the release of cytokines, which can lead to injection reactions (fever, chills, and hemodynamic changes). These reactions occur chiefly during the first few injections and do not contraindicate further rituximab therapy. Serum sickness-like symptoms with the development of antibodies to the chimeric monoclonal antibody are rare and include arthralgia and fever, with onset after the third day. Patients may experience 
persistent hypogammaglobulinemia after rituximab therapy, with an increased risk of infection. Finally, cases of delayed neutropenia and of induced vasculitis have been reported with rituximab.

\subsubsection{Conclusions about rituximab}

Rituximab therapy is not recommended in the situations that were excluded from the pilot study (18), i.e., EGPA, anti-glomerular basement membrane antibody (anti-GBM) disease (Goodpasture syndrome), alveolar hemorrhage requiring mechanical ventilation, and renal failure with a serum creatinine level above $>350 \mu \mathrm{mol} / \mathrm{L}$.

In conclusion, anti-CD20 agents constitute a novel therapeutic approach that is particularly useful for the induction treatment of refractory or relapsing disease, in women of childbearing potential, and for remission maintenance therapy in patients with severe AAV. Anti-CD20 agents will limit the risk of long-term cyclophosphamide-related complications and may diminish the glucocorticoid requirements in patients with AAV, notably GPA. Nevertheless, long-term studies, which are not yet available, must be performed to better define the efficacy and safety of anti-CD20 agents and their effect on management costs in patients with these chronic diseases.

\subsection{Other B-cell targeted therapies}

Other treatment options evaluated in patients with GPA target either the cytokine B lymphocyte stimulator (BLyS) or BAFF. BAFF plays a central role in B-cell selection, differentiation, and homeostasis, and BAFF overexpression may promote the survival of autoreactive B cells. BLyS levels are elevated in patients with AAV, notably those with GPA $(34,35)$. ANCAs can increase the release of BLyS by activated polymorphonuclear cells, 
thereby promoting the survival of activated B cells (35). The monoclonal anti-BLyS antibody belimumab, when evaluated in combination with azathioprine for the remission maintenance treatment of GPA and MPA, failed to lower the relapse rate compared to azathioprine therapy alone (36).

\section{TNF $\alpha$ antagonists}

\subsection{Rationale for using $\mathrm{TNF} \alpha$ antagonists}

Several arguments suggest that targeting $\mathrm{TNF} \alpha$ may provide benefits in patients with AAV. In a murine model, TNF is required to produce an inflammatory response (37). The messenger RNA (mRNA) for TNF is upregulated in mononuclear cells from patients with GPA (38). TNF $\alpha$ promotes the translocation of the PR3 antigen, which is normally located in the cytosol, to the membrane of polymorphonuclear cells, where it is available for binding to ANCA (39). TNF $\alpha$ is produced within extravascular sites involved with GPA (40).

\subsection{Available data on infliximab}

Infliximab is a chimeric monoclonal antibody specifically directed against TNF $\alpha$. In a few patients, a brief course of infliximab was used for the induction treatment of refractory GPA, after failure of conventional therapy, with no concomitant immunosuppressant agent. In open-label studies, which included about 40 patients in all who had failed treatment for GPA, infliximab produced a remission in over $80 \%$ of cases (41). A pilot study performed in France randomly allocated patients with vasculitis refractory to several lines of immunosuppressant therapy to rituximab or infliximab (42). Although a few patients 
responded to infliximab, there was a trend in favor of rituximab, which has now completely superseded infliximab in this indication. Infliximab is also associated with an increased risk of severe infection.

\subsection{Available data on etanercept}

Etanercept is a soluble TNF $\alpha$ receptor. The only randomized controlled trial published to date compared etanercept to a placebo in addition to standard therapy in patients with GPA (43). Etanercept provided no benefits when used for maintenance and the same may apply also to induction therapy. Furthermore, the risk of solid cancer was higher in the group given etanercept versus the placebo, in combination with cyclophosphamide.

\section{Intravenous immunoglobulins}

Immunoglobulins are chiefly composed of human immunoglobulin $\mathrm{G}$ (IgG). Several mechanisms of action have been identified, including neutralization of circulating antibodies; modulation of the complement cascade; and control of B-cell activation, T-cell subsets, dendritic cell function, granulocyte activity, and NK cells. These mechanisms may be interlinked. The therapeutic effect is related to the constant fragment $(\mathrm{Fc})$ and its degree of sialylation, which seems to play a major role. The interaction of the Fc fragment of IgGs with the Fc $\gamma$ receptor on target cells seems crucial, although immunoglobulins also seem to exert a variety of effects on the innate and adaptive immune systems.

Interest in intravenous immunoglobulin therapy (IVIg) for the treatment of AAV arose when studies showed that IVIg prevented the development of coronary aneurysms in patients with Kawasaki disease. In AAV, IVIg has been chiefly used to treat relapses, although 
studies in treatment-naive patients have also been reported $(44,45)$. IVIg therapy in AAV decreases the ANCA titer and is clinically effective in some patients. Tolerance is usually good. Nevertheless, some patients relapse when the IVIg infusions are stopped, limiting the value of this treatment option. IVIg therapy can be particularly helpful when administered in combination with an immunosuppressant to tide a patient with active AAV and severe immunodepression over a difficult period, notably during an infection. IVIg is given either in

a dosage of $2 \mathrm{~g} / \mathrm{kg} / \mathrm{month}$ over 2 days or more gradually in a dose of $0.4 \mathrm{~g} / \mathrm{kg} / \mathrm{day}$ for 5 days, particularly in patients with renal dysfunction. An application for a marketing license has been submitted.

\section{Plasma exchange}

Plasma exchange was first suggested for the treatment of AAV because ANCA was believed to play a pathogenic role. No sound evidence exists to date that plasma exchange may be beneficial as a first-line treatment for AAV in the absence of renal involvement (2). In patients with advanced renal failure, however, plasma exchange is indicated. In a randomized controlled trial, in patients whose baseline serum creatinine level was above 500 $\mu \mathrm{mol} / \mathrm{L}$, plasma exchange was significantly more effective than methylprednisolone boluses in decreasing the dialysis rates after 3 and 12 months (46). However, plasma exchange did not affect overall survival, and the renal function benefits may not be sustained in the long term. Plasma exchange therapy has also been suggested for patients with alveolar hemorrhage, in the absence of scientific evidence to date. An international study in over 700 patients was performed to evaluate the benefits of plasma exchange in patients with moderate renal failure or alveolar hemorrhage 
(https://clinicaltrials.gov/ct2/show/NCT00987389?term=pexivas\&rank=1). The preliminary results will become available in 2018 .

\section{T-cell co-stimulation modulators and T-cell targeted therapies}

Alemtuzumab is a humanized monoclonal antibody to the lymphocyte marker CD52. Although chiefly used in hematological malignancies, alemtuzumab has also been given as rescue therapy to a few patients with very severe and refractory vasculitis (47).

Alemtuzumab induces lymphopenia and is therefore associated with a high risk of infection.

The T-cell co-stimulation inhibitor abatacept produced encouraging results in an open trial. A randomized placebo-controlled trial is under way in patients with relapsing nonsevere GPA (https://clinicaltrials.gov/ct2/show/NCT02108860?term=abrogate\&rank=1).

\section{Eosinophilic granulomatosis with polyangiitis (EGPA, Churg-Strauss syndrome)}

\subsection{Principles of the treatment of eosinophilic granulomatosis with polyangiitis (EGPA)}

EGPA can be treated only at specialized centers. Glucocorticoids remain the mainstay of the treatment of EGPA (2). Immunosuppressants, notably cyclophosphamide, are indicated in patients whose FFS indicates a poor prognosis (Table 1) (5), which is rarely the 
case. In these patients, cyclophosphamide is not indicated as first-line therapy but may be considered if glucocorticoid therapy alone fails or a relapse occurs (2).

\subsection{Rituximab}

Rituximab has been proven beneficial for the induction treatment of severe GPA and MPA in two randomized controlled trials, both of which excluded patients with EGPA. Experience with rituximab in EGPA is therefore too limited to consider this drug as an alternative to first-line cyclophosphamide for the induction treatment of EGPA $(2,15)$.

Several small studies have been reported. They obtained encouraging results with rituximab for induction and maintenance therapy. The largest retrospective study included 41 patients with EGPA, of which one-third had refractory vasculitis and half had relapsing vasculitis (48). The remission rates were 33\% and 50\%, respectively, 6 and 12 months after rituximab initiation. Complete remission after 12 months was significantly more common in patients with than without ANCAs (80\% versus 38\%, respectively). In a limited number of patients with EGPA, another retrospective study recently showed that the relapse-free survival rate was higher with 6-monthly rituximab infusions for maintenance therapy (49).

In France, the randomized controlled double-blind trial REOVAS is comparing rituximab to conventional induction treatment, in combination with standard glucocorticoid therapy, in patients with EGPA (ClinicalTrials.gov NCT02807103). This trial will include 118 patients. Patients with a good prognosis as assessed by the FFS (1) are randomized to rituximab or a placebo and those with cardiac, renal, or gastrointestinal involvement to rituximab or cyclophosphamide. The objective is to determine the efficacy of rituximab in inducing a remission of the vasculitis after 6 months with a prednisone dosage no greater than $\leq 7.5 \mathrm{mg} /$ day. 
The MAINRITSEG double-blind trial is an extension of the above-described trial within the GFEV that compares rituximab to azathioprine for remission maintenance in patients with EGPA (ClinicalTrials.gov Identifier : NCT03164473). Rituximab is given as 500-mg infusions every 6 months for 18 months and azathioprine in a dosage of $2 \mathrm{mg} / \mathrm{kg} / \mathrm{d}$ orally for 24 months. The main outcome measure is the duration of vasculitis remission with a prednisone dosage no greater than $7.5 \mathrm{mg} / \mathrm{d}$.

\subsection{Mepolizumab and other interluekin-5 (IL-5) inhibitors}

Evidence that interleukin-5 (IL-5) plays a key role in the pathogenesis of EGPA by stimulating eosinophil activation and survival has prompted studies of the humanized monoclonal anti-IL-5 antibody mepolizumab as a therapeutic agent. Mepolizumab is licensed for the treatment of severe eosinophilic asthma. For EGPA, the Food and Drug Administration has granted a license extension, and registration of the drug with the European Medicine Agency is under way.

The first two studies of mepolizumab in EGPA were open-label evaluations, each in about 10 patients (50,51). A glucocorticoid-sparing effect was demonstrated, with no vasculitis flares as long as the drug was continued but frequent relapses after it was stopped. The safety profile seems good. An international Phase III randomized controlled trial randomized 136 patients with relapsing or refractory EGPA to either a monthly subcutaneous injection of mepolizumab or a placebo, in addition to standard treatment. Compared to the placebo arm, the mepolizumab arm had a higher remission rate and longer remission duration (including control of the asthma and sinonasal symptoms), as well as lower glucocorticoid requirements (52). Whether the benefits of mepolizumab are due solely to improved control of the asthma and sinonasal symptoms or also to efficacy against the 
extrapulmonary manifestations remains to be evaluated (53). A trial conducted by the GFEV will start soon to evaluate the efficacy of mepolizumab for the induction treatment of EGPA.

Other biological agents targeting either IL-5 (reslizumab) or its receptor (benralizumab) and licensed for use in eosinophilic asthma are being evaluated in patients with EGPA (ClinicalTrials.gov NCT02947945 and NCT03010436) (54).

\subsection{Omalizumab}

Omalizumab is a monoclonal anti-IgE antibody licensed for use in severe persistent allergic asthma. Most of the published data on omalizumab in EGPA come from anecdotal case reports and small retrospective studies, whose results are conflicting. Beneficial effects have been reported in fewer than 30 patients to date. Furthermore, a few case-reports suggest that omalizumab may contribute to trigger vasculitis flares $(55,56)$.

\subsection{Interferon $\alpha(\mathrm{IF} \alpha)$}

Interferon $\alpha$ (IF $\alpha)$ inhibits eosinophil degranulation and effector functions. IF $\alpha$ has been used successfully in a few patients with severe EGPA $(57,58)$. Nevertheless, the available experience is limited, and IF $\alpha$ therapy cannot be recommended until its benefits are validated in a larger number of patients. Cases of apparently noninfectious leukoencephalopathy during IF $\alpha$ therapy have been reported, raising the issue of a possible precipitating role for IF $\alpha(59)$.

\section{6. $\quad \mathrm{TNF} \alpha$ antagonists}


Experience with TNF $\alpha$ antagonists in EGPA is extremely limited. A few patients with extremely severe refractory EGPA despite glucocorticoid and cyclophosphamide therapy showed some measure of responsiveness to TNF $\alpha$ antagonist therapy (60). TNF $\alpha$ antagonists cannot be recommended for EGPA at present.

\section{Intravenous immunoglobulin therapy (IVIg)}

IVIg is sometimes used in patients with EGPA and ANCA antibodies, by analogy with $\mathrm{AAV}$. The total dosage is $2 \mathrm{~g} / \mathrm{kg}$ in two infusions or, particularly in patients with renal dysfunction, over 5 days. Although not recommended as a first-line treatment, IVIg may be useful in combination with other medications in patients who are unresponsive to glucocorticoid and immunosuppressant therapy (refractory vasculitis) and in patients with severe immunodepression, notably those with infections.

\section{Disclosure of interest}

Roche: investigator in studies of vasculitis for which Roche donated part of the rituximab; honoraria for scientific communications;

GSK: investigator in studies of vasculitis for which GSK donated part of the mepolizumab; LFB: honoraria for scientific communications;

Pfizer: honoraria for scientific communications and research grant. 


\section{REFERENCES}

1. Jayne D, Rasmussen N, Andrassy K, et al. A randomized trial of maintenance therapy for vasculitis associated with antineutrophil cytoplasmic autoantibodies. N Engl J Med 2003 ; $349: 36-44$.

2. http://www.hassante.fr/portail/plugins/ModuleXitiKLEE/types/FileDocument/doXiti.jsp?id=c_608140 3. Pagnoux C, Mahr A, Hamidou MA, et al. Azathioprine or methotrexate maintenance for ANCA-associated vasculitis. N Engl J Med $2008 ; 359$ : 2790-803.

4. Puéchal X, Pagnoux C, Perrodeau É, et al. Long-term outcomes among participants in the WEGENT trial of remission-maintenance therapy for granulomatosis with polyangiitis (Wegener's) or microscopic polyangiitis. Arthritis Rheumatol 2016; 68 : 690-701.

5. Guillevin L, Pagnoux C, Seror R, et al. The Five-Factor Score revisited: assessment of prognoses of systemic necrotizing vasculitides based on the French Vasculitis Study Group (FVSG) cohort. Medicine (Baltimore) $2011 ; 90: 19-27$.

6. Ribi C, Cohen P, Pagnoux C, et al. Treatment of polyarteritis nodosa and microscopic polyangiitis without poor-prognosis factors: A prospective randomized study of one hundred twenty-four patients. Arthritis Rheum $2010 ; 62$ : 1186-97.

7. Guillevin L, Cohen P, Mahr A, et al. Treatment of polyarteritis nodosa and microscopic polyangiitis with poor prognosis factors: a prospective trial comparing glucocorticoids and six or twelve cyclophosphamide pulses in sixty-five patients. Arthritis Rheum 2003 ; 49 : 93 100.

8. Krumbholz M, Specks U, Wick M, et al. BAFF is elevated in the serum of patients with Wegener's granulomatosis. J Autoimmun 2005 ; 25 :298-302.

9. Popa ER, Stegeman CA, Bos NA, Kallenberg CG, Tervaert JW. Differential B and T-cell activation in Wegener's granulomatosis. J Allergy Clin Immunol 1999; 103 : 885-94. 
10. Voswinkel J, Müller A, Lamprecht P. Is PR3-ANCA formation initiated in Wegener's granulomatosis lesions? Granulomas as potential lymphoid tissue maintaining autoantibody production. Ann N Y Acad Sci 2005 ; 1051 : 12-9.

11. Voswinkel J, Müller A, Kraemer JA, et al. B lymphocyte maturation in Wegener's granulomatosis: a comparative analysis of VH genes from endonasal lesions. Ann Rheum Dis $2006 ; 65: 859-64$.

12. Jennette JC, Falk RJ. Pathogenesis of antineutrophil cytoplasmic autoantibody-mediated disease. Nat Rev Rheumatol 2014 ; 10 : 463-73.

13. Holden NJ, Williams JM, Morgan MD, et al. ANCA-stimulated neutrophils release BLyS and promote B cell survival: a clinically relevant cellular process. Ann Rheum Dis $2011 ; 70: 2229-33$.

14. Guerry MJ, Brogan P, Bruce IN, et al. Recommendations for the use of rituximab in antineutrophil cytoplasm antibody-associated vasculitis. Rheumatology (Oxford) $2012 ; 51$ : 634-43.

15. http://www.crinet.com/recherche/fichesPratiques/get_fiche.asp?lang=rituximab\&chap=19_Utilisation_du_r ituximab_dans_les_maladies_auto-immunes

16. Holle JU, Dubrau C, Herlyn K, et al. Rituximab for refractory granulomatosis with polyangiitis (Wegener's granulomatosis): comparison of efficacy in granulomatous versus vasculitic manifestations. Ann Rheum Dis 2012 ; 71 : 327-33.

17. Jones RB, Tervaert JW, Hauser T, et al. Rituximab versus cyclophosphamide in ANCAassociated renal vasculitis. N Engl J Med $2010 ; 363: 211-20$.

18. Stone JH, Merkel PA, Spiera R, et al. Rituximab versus cyclophosphamide for ANCAassociated vasculitis. N Engl J Med $2010 ; 363: 221-32$. 
19. Miloslavsky EM, Specks U, Merkel PA, et al. Clinical outcomes of remission induction therapy for severe antineutrophil cytoplasmic antibody-associated vasculitis. Arthritis Rheum 2013 ; 65 : 2441-9.

20. Unizony S, Villarreal M, Miloslavsky EM, et al. Clinical outcomes of treatment of antineutrophil cytoplasmic antibody (ANCA)-associated vasculitis based on ANCA type. Ann Rheum Dis 2016 ; 75 : 1166-9.

21. Specks U, Merkel PA, Seo P, et al. Efficacy of remission-induction regimens for ANCAassociated vasculitis. N Engl J Med $2013 ; 369$ : 417-27.

22. Miloslavsky EM, Specks U, Merkel PA, et al. Rituximab for the treatment of relapses in antineutrophil cytoplasmic antibody-associated vasculitis. Rituximab in ANCA-Associated Vasculitis-Immune Tolerance Network Research Group. Arthritis Rheumatol 2014 ; 66 : 3151-9.

23. Smith R, Jones R, Specks U, et al. Rituximab as re-induction therapy in relapsing ANCA-associated vasculitis [abstract]. Arthritis Rheumatol 2017; 69 (suppl 10) : 18L. 24. Puéchal X, Iudici M, Calich AL, et al. Rituximab for induction and maintenance therapy of granulomatosis with polyangiitis: a single-centre cohort study on 114 patients. Rheumatology (Oxford) 2018. doi: 10.1093/rheumatology/key117. 25. Smith RM, Jones RB, Guerry MJ, et al. Rituximab for remission maintenance in relapsing ANCA-associated vasculitis. Arthritis Rheum 2012 ; 64 : 3760-9.

26. Azar L, Springer J, Langford CA, Hoffman GS. Rituximab with or without a conventional maintenance agent in the treatment of relapsing granulomatosis with polyangiitis (Wegener's): a retrospective single-center study. Arthritis Rheumatol 2014 ; 66 : 2862-70. 
27. Guillevin L, Pagnoux C, Karras A, et al for the French Vasculitis Study Group (FVSG): Rituximab versus azathioprine for maintenance in ANCA-associated vasculitis. N Engl J Med $2014: 371: 1771-80$.

28. Terrier B, Pagnoux C, Perrodeau É, et al. Long-term efficacy of remission-maintenance regimens for ANCA-associated vasculitides. Ann Rheum Dis 2018;77:1150-6.

29. Cartin-Ceba R, Golbin JM, Keogh KA, et al. Rituximab for remission induction and maintenance in refractory granulomatosis with polyangiitis (Wegener's): A single-center tenyear experience. Arthritis Rheum $2012 ; 64: 3770-8$.

30. Charles P, Terrier B, Perrodeau É, et al. Comparison of individually tailored versus fixed-schedule rituximab regimen to maintain ANCA-associated vasculitis remission: results of a multicentre, randomised controlled, phase III trial (MAINRITSAN2). Ann Rheum Dis 2018;77:1143-9.

31. Charles P, Bienvenu B, Bonnotte B, et al. Rituximab: Recommendations of the French Vasculitis Study Group (FVSG) for induction and maintenance treatments of adult, antineutrophil cytoplasm antibody-associated necrotizing vasculitides. Presse Med 2013 ; $42: 1317-30$.

32. Molloy ES, Calabrese LH. Progressive multifocal leukoencephalopathy associated with immunosuppressive therapy in rheumatic diseases: Evolving role of biologic therapies. Arthritis Rheum 2012 ; 64 : 3043-51.

33. van Daalen EE, Rizzo R, Kronbichler A, et al. Effect of rituximab on malignancy risk in patients with ANCA-associated vasculitis. Ann Rheum Dis 2017 ; 76 : 1064-9.

34. Schneeweis C, Rafalowicz M, Feist E, et al. Increased levels of BLyS and sVCAM-1 in anti-neutrophil cytoplasmatic antibody (ANCA)-associated vasculitides (AAV). Clin Exp Rheumatol 2010 ; 28 (1 Suppl 57) : 62-6. 
35. Holden NJ, Williams JM, Morgan MD, et al. ANCA-stimulated neutrophils release BLyS and promote B cell survival: a clinically relevant cellular process. Ann Rheum Dis $2011 ; 70: 2229-33$.

36. Jayne D, Blockmans D, Luqmani R, et al. Efficacy and safety of belimumab in combination with azathioprine for remission maintenance in granulomatosis with polyangiitis and microscopic polyangiitis: a multicenter randomized, placebo-controlled study [abstract]. Arthritis Rheumatol 2017; 69 (suppl 10): 893.

37. Pfister H, Ollert M, Fröhlich LF, et al. Antineutrophil cytoplasmic autoantibodies against the murine homolog of proteinase 3 (Wegener autoantigen) are pathogenic in vivo. Blood $2004 ; 104: 1411-8$.

38. Deguchi Y, Shibata N, Kishimoto S. Enhanced expression of the tumour necrosis factor/cachectin gene in peripheral blood mononuclear cells from patients with systemic vasculitis. Clin Exp Immunol $1990 ; 81$ : 311-4.

39. Csernok E, Ernst M, Schmitt W, et al. Activated neutrophils express proteinase 3 on their plasma membrane in vitro and in vivo. Clin Exp Immunol 1994 ; 95 : 244-5.

40. Noronha IL, Krüger C, Andrassy K, Ritz E, Waldherr R. In situ production of TNFalpha, IL-1 beta and IL-2R in ANCA-positive glomerulonephritis. Kidney Int 1993 ; 43 : $682-92$

41. Puéchal X, Guillevin L. Therapeutic immunomodulation in systemic vasculitis: taking stock. Joint Bone Spine $2013 ; 80$ : 374-9.

42. de Menthon M, Cohen P, Pagnoux C, et al. Infliximab or rituximab for refractory Wegener's granulomatosis: long-term follow up. A prospective randomised multicentre study on 17 patients. Clin Exp Rheumatol 2011 ; 29 (1 Suppl 64) : S63-71.

43. The Wegener's Granulomatosis Etanercept Trial (WGET) Research Group : etanercept plus standard therapy for Wegener's granulomatosis. N Engl J Med 2005 ; 352 : 351-61. 
44. Jayne DR, Chapel H, Adu D, et al. Intravenous immunoglobulin for ANCA-associated systemic vasculitis with persistent disease activity. Q J Med 2000 ; 93 : 433-9.

45. Martinez V, Cohen P, Pagnoux C, et al. Intravenous immunoglobulins for relapses of systemic vasculitides associated with antineutrophil cytoplasmic autoantibodies: results of a multicenter, prospective, open-label study of twenty-two patients. Arthritis Rheum 2008 ; $58: 308-17$.

46. Jayne DR, Gaskin G, Rasmussen R, et al. Randomized trial of plasma exchange or highdosage methylprednisolone as adjunctive therapy for severe renal vasculitis. J Am Soc Nephrol $2007 ; 18: 2180-8$.

47. Lockwood C, Thiru S, Isaacs J, et al. Long-term remission of intractable systemic vasculitis with monoclonal antibody therapy. Lancet $1993 ; 1$ : 1620-2.

48. Mohammad AJ, Hot A, Arndt F, et al. Rituximab for the treatment of eosinophilic granulomatosis with polyangiitis (Churg-Strauss). Ann Rheum Dis 2016 ; 75 : 396-401. 49. Emmi G, Rossi GM, Urban ML, et al. Scheduled rituximab maintenance reduces relapse rate in eosinophilic granulomatosis with polyangiitis. Ann Rheum Dis 2018;77:952-4. 50. Kim S, Marigowda G, Oren E, et al. Mepolizumab as a steroid-sparing treatment option in patients with Churg-Strauss syndrome. J Allergy Clin Immunol $2010 ; 125$ : 1336-43. 51. Moosig F, Gross WL, Herrmann K, et al. Targeting interleukin-5 in refractory and relapsing Churg-Strauss syndrome. Ann Intern Med $2011 ; 155$ : 341-3.

52. Wechsler ME, Akuthota P, Jayne D, et al. Mepolizumab or placebo for eosinophilic granulomatosis with polyangiitis. N Engl J Med 2017 ; 376 : 1921-32.

53. Guillevin L. Vasculitis: Mepolizumab for eosinophilic granulomatosis with polyangiitis. Nat Rev Rheumatol 2017 ; 13 : 518-9.

54. Puéchal X. Perspectives de traitement par biomédicaments dans la granulomatose éosinophilique avec polyangéite (Churg-Strauss). Revue Rhum 2017 ; 84 : 256-61. 
55. Puéchal X, Rivereau P, Vinchon F. Churg Strauss syndrome associated with omalizumab. Eur J Intern Med 2008 ; 19 : 364-6.

56. Jachiet M, Samson M, Cottin V, et al. Anti-IgE monoclonal antibody (omalizumab) in refractory and relapsing eosinophilic granulomatosis with polyangiitis (Churg-Strauss): Data on seventeen patients. Arthritis Rheumatol 2016 ; 68 : 2274-82.

57. Tatsis E, Schnabel A, Gross WL. Interferon-alpha treatment of four patients with the Churg-Strauss syndrome. Ann Intern Med $1998 ; 129: 370-4$.

58. Metzler C, Schnabel A, Gross WL, Hellmich B. A phase II study of interferon-alpha for the treatment of refractory Churg-Strauss syndrome. Clin Exp Rheumatol 2008 ; 26 (3 Suppl 49): S35-40.

59. Metzler C, Lamprecht P, Hellmich B, Reuter M, Arlt AC, Gross WL. Leucoencephalopathy after treatment of Churg-Strauss syndrome with interferon alpha. Ann Rheum Dis 2005 ; 64 : 1242-3.

60. Arbach O, Gross WL, Gause A. Treatment of refractory Churg-Strauss-Syndrome (CSS) by TNF-alpha blockade. Immunobiology $2002 ; 206: 496-501$. 
Table 1. Five-Factor Score evaluating factors of adverse prognostic significance in patients with microscopic polyangiitis, granulomatosis with polyangiitis (Wegener), and eosinophilic granulomatosis with polyangiitis (Churg-Strauss) (5)

- age older than 65 years

- serum creatinine $>150 \mu \mathrm{mol} / \mathrm{L}$

- specific cardiomyopathy

- specific gastrointestinal involvement

- absence of ENT involvement in a patient diagnosed with granulomatosis with polyangiitis

(Wegener) or eosinophilic granulomatosis with polyangiitis (Churg-Strauss)

The prognostic Five-Factor Score (FFS) is 0 when all five factors are absent and equals 1 when a single factor is present, 2 when two factors are present, and so on. 